\title{
Discovery of off-axis jet structure of TeV blazar Mrk 501 with $\mathrm{mm}-\mathrm{VLBI}$
}

\author{
S. Koyama ${ }^{1}$, M. Kino ${ }^{2}$, M. Giroletti ${ }^{3}$, A. Doi ${ }^{4}$, G. Giovannini ${ }^{3,5}$, M. Orienti ${ }^{3}$, K. Hada $^{3,6}$, \\ E. Ros ${ }^{1,7,8}$, K. Niinuma ${ }^{9}$, H. Nagai ${ }^{6}$, T. Savolainen ${ }^{1,10}$, T. P. Krichbaum ${ }^{1}$, and M. Á. Pérez-Torres ${ }^{11,12}$ \\ ${ }^{1}$ Max-Planck-Institut für Radioastronomie, Auf dem Hügel 69, 53121 Bonn, Germany \\ e-mail: skoyama@mpifr-bonn.mpg.de \\ 2 Korea Astronomy and Space Science Institute, 776 Daedeokdae-ro, Yuseong-gu, 305-348 Daejeon, Republic of Korea \\ 3 INAF-Istituto di Radioastronomia, via Gobetti 101, 40129 Bologna, Italy \\ ${ }^{4}$ Institute of Space and Astronautical Science, Japan Aerospace Exploration Agency, 3-1-1 Yoshinodai, Chuou-ku, Sagamihara, \\ 252-5210 Kanagawa, Japan \\ 5 Dipartimento di Astronomia, Università di Bologna, via Ranzani 1, 40127 Bologna, Italy \\ 6 National Astronomical Observatory of Japan, 2-21-1 Osawa, Mitaka, 181-8588 Tokyo, Japan \\ 7 Observatori Astronòmic, Universitat de València, 46980 Paterna, València, Spain \\ 8 Departament d'Astronomia i Astrofísica, Universitat de València, 46100 Burjassot, València, Spain \\ 9 Graduate School of Science and Engineering, Yamaguchi University, 753-8511 Yamaguchi, Japan \\ 10 Aalto University Metsähovi Radio Observatory, 02540 Kylmälä, Finland \\ 11 Instituto de Astrofísica de Andalucía, Glorieta de la Astronomía, s/n, 18008 Granada, Spain \\ 12 Centro de Estudios de la Física del Cosmos de Aragón, 44001 Teruel, Spain
}

Received 17 May 2015 / Accepted 7 December 2015

\section{ABSTRACT}

\begin{abstract}
Context. High-resolution millimeter wave very-long-baseline interferometry (mm-VLBI) is an ideal tool for probing the structure at the base of extragalactic jets in detail. The TeV blazar Mrk 501 is one of the best targets among BL Lac objects for studying the nature of off-axis jet structures because it shows different jet position angles at different scales.

Aims. The aim of this study is to investigate the properties of the off-axis jet structure through high-resolution mm-VLBI images at the jet base and physical parameters such as kinematics, flux densities, and spectral indices.

Methods. We performed Very Long Baseline Array (VLBA) observations over six epochs from 2012 February to 2013 February at $43 \mathrm{GHz}$. Quasi-simultaneous Global Millimeter VLBI Array (GMVA) observations at $86 \mathrm{GHz}$ were performed in May 2012.

Results. We discover a new jet component at the northeast direction from the core in all the images at 43 and $86 \mathrm{GHz}$. The new component shows the off-axis location from the persistent jet extending to the southeast. The $43 \mathrm{GHz}$ images reveal that the scattering of the positions of the NE component is within $\sim 0.2$ mas. The $86 \mathrm{GHz}$ data reveals a jet component located 0.75 mas southeast of the radio core. We also discuss the spectral indices between 43 and $86 \mathrm{GHz}$, where the northeast component has steeper spectral index and the southeast component has comparable or flatter index than the radio core does.
\end{abstract}

Key words. BL Lacertae objects: individual: Markarian 501 - galaxies: active - galaxies: jets - radio continuum: galaxies

\section{Introduction}

Relativistic outflows (jets) in active galactic nuclei (AGNs) are ultimately powered by gravitational energy of supermassive black holes lying in the center of galaxies (e.g., Begelman et al. 1984). Probing structures and kinematics of the inner parts of jets is a fundamental issue for understanding jet physics. The intensive systematic Very Long Baseline Array (VLBA) monitoring of relativistic outflows in a sample of over 100 AGN jets at $15 \mathrm{GHz}$ has been conducted by the Monitoring Of Jets in Active galactic nuclei with VLBA Experiments (MOJAVE) project (e.g., Kellermann et al. 1998; Lister et al. 2009, 2013; Homan et al. 2009, 2015). The MOJAVE data have revealed that a large fraction of jets at milliarcsecond scales show evidence of curved motion.

Recently, a few very-long-baseline interferometry (VLBI) observations at frequencies higher than $43 \mathrm{GHz}$ have revealed the inner structure of the curved jets and their kinematics at submilliarcsecond scales. Monthly monitoring of blazars with the VLBA at $43 \mathrm{GHz}$ reveals large swings of the jet position angle (PA) in their innermost regions (e.g., OJ 287: Agudo et al. 2012, as a part of the Boston University Blazar Project). Lu et al. (2013) find an unusual jet PA in the quasar 3C 279 by combining Event Horizon Telescope observations at $230 \mathrm{GHz}$ and VLBA observations at $43 \mathrm{GHz}$ at $\sim 0.2$ mas $(\sim 1 \mathrm{pc})$ scales. The new jet PA is almost perpendicular to the persistent jet axis at a scale of a few pc observed at or lower than $43 \mathrm{GHz}$ (hereafter called "offaxis jet structure"). Thus, high-resolution VLBI observations at high frequencies $(\geq 43 \mathrm{GHz})$ are important to explore such offaxis jet structures. Off-axis jet structures at the base of radio jets could be related to the properties of the jet formation regions; however, their nature is still poorly understood.

The TeV blazar Mrk 501 is one of the best BL Lac objects for studying the innermost jet thanks to its proximity $(z=0.034)$ and brightness. The jet PAs of this source show different apparent directions on different angular scales, changing smoothly in counter-clockwise direction. Low-frequency observations have revealed that the jet PA (defined positive north through east) 
Table 1. Journal of VLBI observations.

\begin{tabular}{|c|c|c|c|c|c|c|c|c|}
\hline Epoch & $\begin{array}{l}\text { Observing } \\
\text { code }\end{array}$ & $\begin{array}{c}\text { Frequency } \\
(\mathrm{GHz})\end{array}$ & $\begin{array}{c}\text { Map peak } \\
\left(\mathrm{mJy}^{-} \text {beam }^{-1}\right)\end{array}$ & (mas) & $\begin{array}{l}\text { eam size } \\
\text { (mas) }\end{array}$ & (deg) & $\begin{array}{c}1 \sigma \\
\left(\mathrm{mJy} \mathrm{beam}^{-1}\right)\end{array}$ & Notes \\
\hline 2012 Feb.12 & BK172A & 43 & 209 & 0.46 & 0.12 & -22.4 & 1.25 & no KP, SC, FD \\
\hline 2012 Mar.16 & BK172B & 43 & 165 & 0.33 & 0.12 & -15.0 & 0.98 & \\
\hline 2012 May 06 & BK172C & 43 & 215 & 0.40 & 0.12 & -17.2 & 1.30 & $\mathrm{OV}, \mathrm{SC}^{b}$ \\
\hline 2012 Jun.11 & BK172D & 43 & 267 & 0.35 & 0.17 & 12.8 & 0.96 & no $\mathrm{MK}$ \\
\hline 2013 Jan.18 & BK172E & 43 & 148 & 0.35 & 0.13 & -19.6 & 0.85 & $\mathrm{HN}^{b}$ \\
\hline 2013 Feb. 15 & BK172F & 43 & 144 & 0.33 & 0.12 & -22.0 & 1.06 & $\mathrm{FD}, \mathrm{PT}^{b}$ \\
\hline 2012 Мay 19 & GG072 & 86 & 99 & 0.21 & 0.04 & -5.11 & 1.93 & see details in Sect. 2.2 \\
\hline
\end{tabular}

Notes. ${ }^{(a)}$ Major axis, minor axis, and position angle of synthesized beam. ${ }^{(b)}$ The stations were affected by significant weather or technical problems.
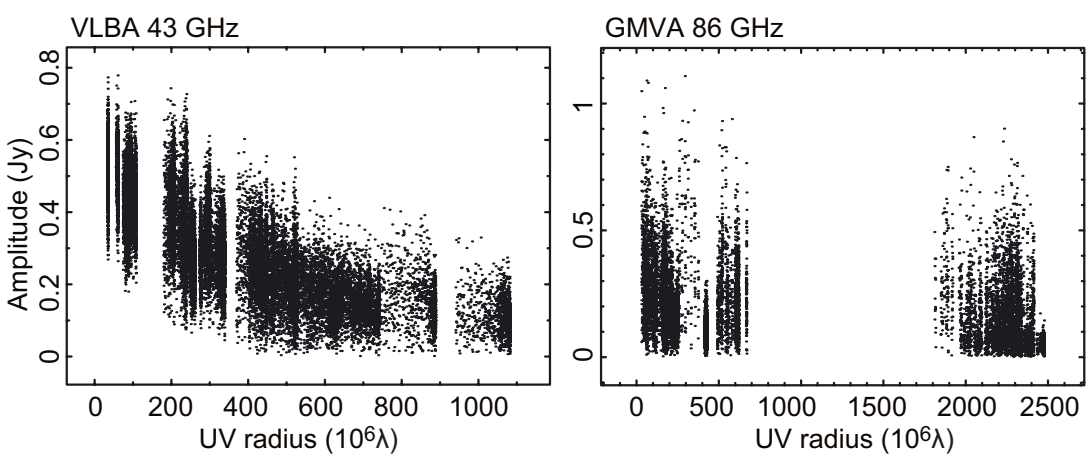

Fig. 1. Visibility amplitude vs. $(u, v)$-radius for the third epoch VLBA observations at $43 \mathrm{GHz}$ (left panel) and for the GMVA observations at $86 \mathrm{GHz}$ (right panel).

is $\sim 45^{\circ}$ at $r>20$ mas from the core, and $\sim 100^{\circ}$ at $2<r<$ 20 mas (Giroletti et al. 2004, 2008). VLBA images at $43 \mathrm{GHz}$ have shown a clear limb-brightened structure toward PA $\sim 150^{\circ}$ (labeled eastern limb) and PA $\sim 175^{\circ}$ (labeled western limb) at $0.5<r<2$ mas (Piner et al. 2009, 2010). The Global Millimeter VLBI Array (GMVA) observations at $86 \mathrm{GHz}$ show a jet feature toward $\mathrm{PA} \sim 172^{\circ}$ at 0.73 mas and the inner 0.1 mas jet limbs at $\mathrm{PA} \sim-135^{\circ}$ and $\mathrm{PA} \sim 144^{\circ}$ (Giroletti et al. 2008).

In this paper, we report on the sub-pc to pc scale structure of Mrk 501 obtained with VLBA $43 \mathrm{GHz}$ and GMVA $86 \mathrm{GHz}$ observations in 2012-2013, focusing on a newly found off-axis jet structure. In Sect. 2 we describe the observations; in Sect. 3 we present the results; and in Sect. 4 we give a discussion of our findings. Throughout this paper, we adopt the following cosmological parameters: $H_{0}=71 \mathrm{~km} \mathrm{~s}^{-1} \mathrm{Mpc}^{-1}, \Omega_{\mathrm{M}}=0.27$, and $\Omega_{\Lambda}=0.73$ (Komatsu et al. 2009), or 1 mas $=0.66 \mathrm{pc}$ for Mrk 501.

\section{Observations and data reductions}

\subsection{VLBA}

In Table 1, we summarize the details of our VLBI observations. Six epochs of observations were carried out at $43 \mathrm{GHz}$ between 2012 February and 2013 February with ten VLBA stations: Kitt Peak (KP), Saint Croix (SC), Fort Davis (FD), Owens Valley (OV), Mauna Kea (MK), Hancock (HN), Pie Town (PT), North Liberty (NL), Brewster (BR), and Los Alamos (LA). The central frequency was $43.212 \mathrm{GHz}$, with eight subbands (IFs) of $16 \mathrm{MHz}$ bandwidth each. Left hand circular polarization (LHCP) was recorded at a bit-rate of $512 \mathrm{Mbps}$ by Mark 5 disk systems, and was correlated at the National Radio Astronomy Observatory (NRAO) VLBA-DiFX software correlator (Deller et al. 2011). In some epochs, one or more antennas did not work properly because of technical problems or weather condition (see Table 1). For each epoch, the total onsource time for Mrk 501 was approximately one hour, distributed over a four-hour long track.

Initial data calibration was performed using the Astronomical Image Processing System ( $\mathcal{A} \mathcal{I P S}$ ) software package developed by the NRAO. A priori amplitude calibration was done based on the measurements of the system noise temperature during the observations and the elevation-dependent antenna gain provided by each station. In this process, we also applied opacity correction due to the atmospheric attenuation, assuming that the time variation of the opacity is not significant during each observation. Phase and delay offset between different sub-bands were solved by using the calibrator 3C 345. Fringe fitting was performed with the $\mathcal{A I P S}$ task FRING on Mrk 501 by averaging over all the IFs. Imaging and self-calibration were performed using the Difmap software package (Shepherd 1997). The final images were produced after iterations of CLEAN, phase, and amplitude self-calibration processes.

The $(u, v)$ range is $32-1082 \mathrm{M} \lambda$ for the third epoch as an example (Fig. 1 left), and its maximum is $1242 \mathrm{M} \lambda$ for the second epoch.

\subsection{GMVA}

We observed Mrk 501 on 2012 May 19 with the GMVA at $86 \mathrm{GHz}$, which was 13 days after the third epoch of VLBA $43 \mathrm{GHz}$ observations. The central frequency was $86.198 \mathrm{GHz}$, with eight IFs of $16 \mathrm{MHz}$ bandwidth each. The LHCP was recorded at a bit-rate of 512 Mbps by Mark 5 disk systems, and was correlated at the Bonn DiFX software correlator. 
The participating instruments were four European telescopes (Effelsberg (EF), the Plateau de Bure interferometer (PB), Onsala (ON), and Yebes (YB)), and seven VLBA stations (all except SC, HN, and MK). We observed the calibrator 3C 345 and the target with a time cycle of $16 \mathrm{~min}$, where two minutes were allocated for calibrator 3C 345, five minutes for target Mrk 501, and the remaining time for antenna pointing and calibration. The total observation time was approximately $12 \mathrm{~h}$ (UT 0/20:00-1/08:30). The European telescopes observed for the first $\sim 9 \mathrm{~h}$, and the VLBA joined in for the last $\sim 6 \mathrm{hr}$. The telescopes at $\mathrm{EF}$ and $\mathrm{OV}$ failed owing to system problems (low signal-tonoise ratio $(\mathrm{S} / \mathrm{N})$, and power supply problems, respectively).

The calibrator 3C 345 was detected with a $S / N>7$ on all baselines. From the fringe fitting of 3 C 345 , we determined single-band delay offsets using good scans and applied them to the whole data set. We performed global fringe fitting on 3C 345 over all the IFs to determine the phase, delay, and rate, using a solution interval of two minutes. CLEAN and phase self-calibration were performed iteratively, and one amplitude self-calibration for 3C 345 was applied using the GSCALE command of Difmap. We obtained an image of 3C 345 and found the structure within 2 mas from the core to be in agreement with the archival $43 \mathrm{GHz}$ VLBA image taken around a week after our GMVA session (on 2012 May 27) ${ }^{1}$. The total GMVA $86 \mathrm{GHz}$ cleaned flux of 3C 345 was $1.7 \mathrm{Jy}$, which is approximately $50 \%$ of the single-dish flux at $86 \mathrm{GHz}^{2}$. Since there are only small differences $(\sim 5 \%)$ between the single-dish fluxes and the total VLBA fluxes at $15 \mathrm{GHz}^{3}$ and $43 \mathrm{GHz}^{1}$, we attribute this much larger difference to a significant flux loss for the GMVA $86 \mathrm{GHz}$. Such flux losses can sometimes be seen in the images obtained with GMVA at $86 \mathrm{GHz}$ (e.g., Rani et al. 2015). The possible reasons could be antenna pointing error, unmodeled antenna efficiency, and missing flux from extended structure. To derive a scaling factor of 3C 345 for the GMVA $86 \mathrm{GHz}$, we estimated the extended flux at $15 \mathrm{GHz}$ and $43 \mathrm{GHz}$ as the difference between the single-dish fluxes and the total cleaned VLBI fluxes, and extrapolated the value of the extended flux at $86 \mathrm{GHz}$ by using the spectral index $(\alpha \sim-0.6$ as determined between $15 \mathrm{GHz}$ and $43 \mathrm{GHz}$ ). We then estimated the expected GMVA $86 \mathrm{GHz}$ flux as $\sim 3.5 \mathrm{Jy}$, and determined the scaling factor to be around 2.1 for 3 C 345 . Since the source compactness varies between sources (e.g., Lee et al. 2008), we give the estimate of the scaling factor for Mrk 501 at the end of this section.

At this stage, it was then possible to fringe fit Mrk 501 itself using the fringe solutions of 3C 345, averaging over all the IFs. For fringe fitting, we used a solution interval of five minutes because the $\mathrm{S} / \mathrm{N}$ of the solutions increased as we increased the solution intervals by $0.5 \mathrm{~min}(0.5-5 \mathrm{~min})$. Since $3 \mathrm{C} 345$ is close to Mrk 501 (separation of $2.09^{\circ}$ ), most of the phase variations were corrected by $3 \mathrm{C} 345$ and the residual delay and phase rate for Mrk 501 became quite small. Therefore, we set a small search window of a few tens of nsec and $\mathrm{mHz}$ in delay and rate, respectively, with a low $\mathrm{S} / \mathrm{N}$ threshold. The overall $(u, v)$ range is $29-2476 \mathrm{M} \lambda$ with sparse coverage in the interval 668$1809 \mathrm{M} \lambda$ (Fig. 1 right). After some iterations of fringe fitting, we find smooth delay and phase rate solutions clustering each other in the following conditions. Fringes were found between European stations (setting $\mathrm{S} / \mathrm{N}$ cutoff 2.8 , maximum $S / N \sim 7.0$ )

\footnotetext{
1 http://www.bu.edu/blazars/VLBAproject.html

2 http://www3.mpifr-bonn.mpg.de/div/vlbi/fgamma/ results.html

3 http://WWW . physics . purdue.edu/astro/MOJAVE/ allsources.html
}

and between VLBA telescopes (setting $\mathrm{S} / \mathrm{N}$ cutoff 2.5, maximum $S / N \sim 6.0$ ). Fringes of transatlantic baselines were only detected between $\mathrm{PB}$ and $\mathrm{KP}$, and $\mathrm{PB}$ and LA, during several good scans (setting $\mathrm{S} / \mathrm{N}$ cutoff 2.5, maximum $S / N \sim 3.0$ ). Although the solutions on the long baselines might be less significant (they may lead to the overestimation of the correlated flux and compactness), the emission on the short baselines is clearly detected. In any case, we edited out the solutions that were obviously bad with the $\mathcal{A I P S}$ task SNEDT and we subsequently frequency averaged the data. Gaussian model fitting and phase self-calibration were performed in Difmap. Owing to the low $\mathrm{S} / \mathrm{N}$ of the visibilities, we did not perform amplitude self-calibration.

We then derived the scaling factor for the GMVA image of Mrk 501. The total modeled flux of Mrk 501 by the GMVA at $86 \mathrm{GHz}$ was $286 \mathrm{mJy}$, which is approximately $40 \%$ of the singledish flux $(\sim 750 \mathrm{mJy})$ at $86 \mathrm{GHz}^{2}$, obtained around a week before the GMVA observations. On the other hand, at $15 \mathrm{GHz}^{3}$ and $43 \mathrm{GHz}^{1}$, the total cleaned VLBI fluxes are around $60 \%$ of the single-dish measurement, such that there might be some flux loss for the GMVA flux. We derived the spectral index of the extended flux to be $\alpha \sim-0.4$ in the same manner as 3C 345, then estimated the expected GMVA $86 \mathrm{GHz}$ flux as $\sim 510 \mathrm{mJy}$, and finally determined the scaling factor to be around 1.8 for Mrk 501.

\section{Results}

\subsection{VLBA results}

\subsubsection{Images}

Figure 2 shows the total-intensity CLEAN images obtained with the VLBA at $43 \mathrm{GHz}$ as contours. The rms noise level on our images is approximately $1 \mathrm{mJy}^{\text {beam }}{ }^{-1}$, a factor of a few times higher than the noise level expected theoretically $\left(0.32 \sim 0.47 \mathrm{mJy} \mathrm{beam}^{-1}\right.$ estimated from VLBA observational status summary). The rms level is comparable to or slightly higher than the noise level on the images of Mrk 501 in Piner et al. (2009), whose observing frequency, aperture, settings, and on-source time (40 min) are similar to ours.

All the images show a bright core and a one-sided jet structure elongated toward the southeast. The core and one-sided limb-brightened southeast jet have been observed in previous images, and we give a detailed comparison in Sect. 3.1.3. Our most remarkable finding is the emergence of new emission toward the northeast and to the east of the core in all six images. Compared to the dense contours west of the core, the contours east of the core are clearly sparse and indicate the presence of emission from an additional component. We searched the website of Boston University's blazar monitoring program ${ }^{1}$ for images of similar quality. All the images available between 2012 October 19 and 2014 December 5 are in good agreement with our images, showing emission elongated toward the northeast to the east of the core. In the VLBA $43 \mathrm{GHz}$ image obtained on 2011 September 24, the northeast emission was not seen, possibly due to the lack of dynamic range.

Piner et al. (2009, 2010) presented VLBA $43 \mathrm{GHz}$ images between 2005 and 2009 of a quality comparable to ours; however, clear emission elongated northeast of the core was not detected. Therefore, the northeast emission has emerged at least since the first epoch of our observations (2012 February 12), and the emergence might be related to a $\gamma$-ray flare in 2011 October (Bartoli et al. 2012). 

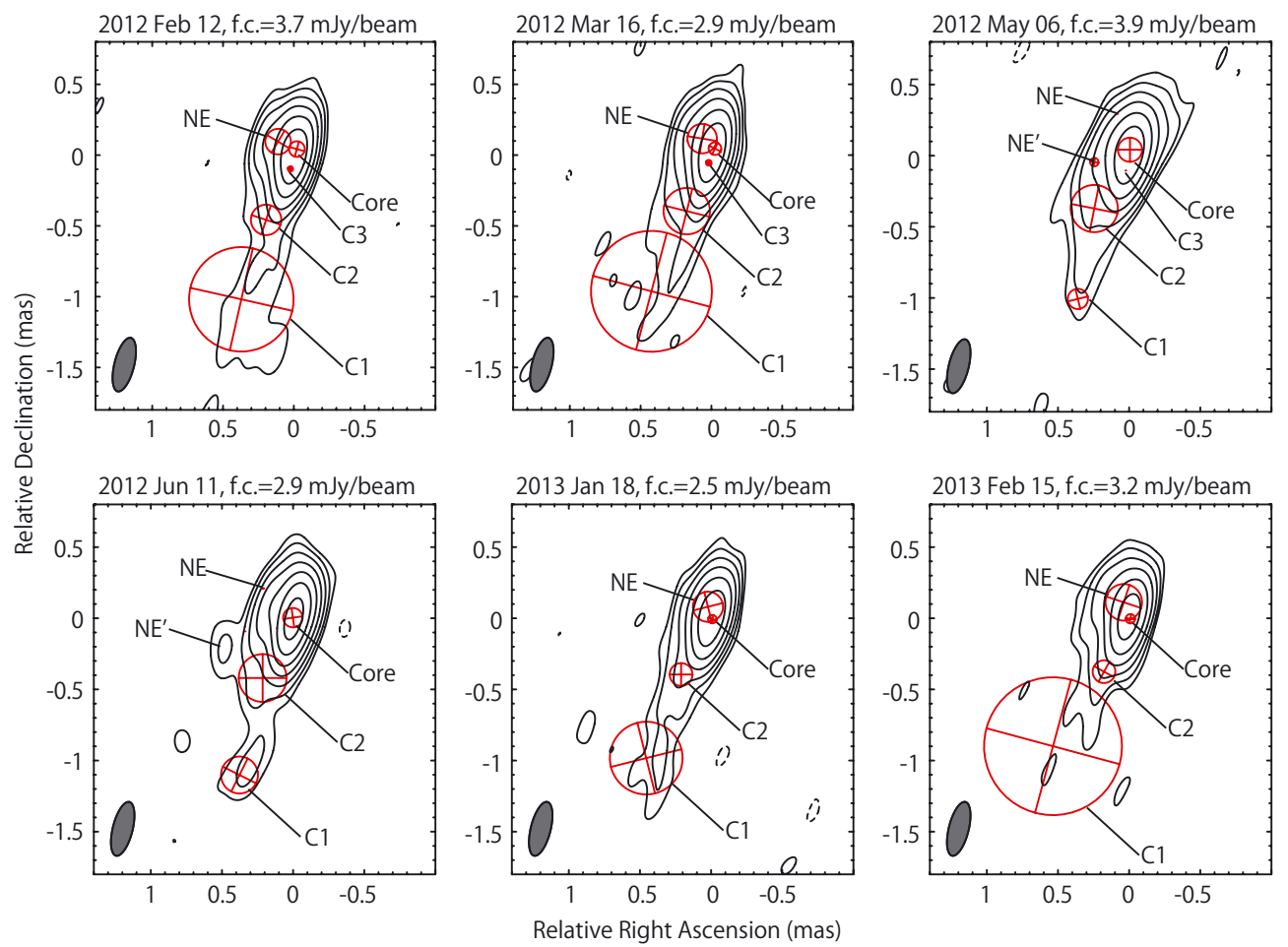

Fig. 2. Uniform weighted VLBA $43 \mathrm{GHz}$ CLEAN images with fitted circular Gaussian components. The restoring beam is 0.39 mas $\times 0.14$ mas in $\mathrm{PA}=-14^{\circ}$, plotted in the bottom left corner. Observing date and the first contour (f.c.) are plotted above each map. The first contours are all set to three times the rms noise level of each map, increasing by a factor of 2 .

\subsubsection{Model fitting}

In order to parametrize the brightness distribution of the jet, we used the task MODELFIT in Difmap. In the model-fit process, we used a set of circular Gaussian model components to fit the visibility data in the $(u, v)$ plane. In Fig. 2, the model components are overlaid on the CLEAN images. Table 2 lists the resulting model-fit parameters at every observing epoch.

Overall structures are mostly represented by four to six model components. We note that without a model component northeast of the core, there were brighter residuals at the northeast of the core than at the southeast of the core. For the one month separation data, i.e. between 2012 February and March, between 2012 May and June, and between 2013 January and February, we repeated the model fitting of the latter epochs using the model components from the former epochs. For the data with longer than two months separation, we performed the model fitting procedure independently due to the possible structural changes. In the component identification process, we identified the brightest, most compact, and innermost feature as the radio core, and the northeast component as NE. Hereafter, the other components are as follows: $\mathrm{C} 1$ as the outermost component, $\mathrm{C} 2$ as the second outermost component, and $\mathrm{C} 3$ as the third outermost jet component toward the southeast. The jet extends to roughly 1.1 mas southeast of the core. In the third and fourth epoch images, we find the component labeled $\mathrm{NE}^{\prime}$, which elongates southeast of the NE component.

The uncertainties of the model-fit parameters presented in Table 2 are estimated by following the methods described in Lico et al. (2012) and Blasi et al. (2013). The position errors of each component are estimated using the ratio of the size of each component to the $\mathrm{S} / \mathrm{N}$. However, most of the components are smaller than the beam size of the image, such that the position errors are replaced by a conservative value equal to $10 \%$ of the beam size (Lister et al. 2009; Orienti et al. 2011). The flux density uncertainties are estimated considering the addition in quadrature of a calibration error of about $10 \%$ and a statistical error given by three times the rms noise of the map.

\subsubsection{Angular separation and position angle of individual components}

Figures 3 and 4 show the angular separation and the position angle of each component relative to the core. The components $\mathrm{C} 1$ and $\mathrm{C} 2$ are consistent with being quasi-stationary, since there is no significant proper motion by means of linear fits to the separation from the core over more than three epochs $(0.02 \pm 0.15 c$ for the component $\mathrm{C} 1$ and $-0.10 \pm 0.09 c$ for the component $\mathrm{C} 2$ ). We compare the locations of the components $\mathrm{C} 1, \mathrm{C} 2$, and $\mathrm{C} 3$ (hereafter the southeast jet) to those in previous studies. Piner et al. (2009) find that VLBA images at $43 \mathrm{GHz}$ in 2005 have shown a clear limb-brightened structure to the southeast, which is labeled as the eastern limb (PA $\sim 150^{\circ}$ ) and western limb (PA $\sim 175^{\circ}$ ) at $0.5<r<2$ mas using the model fitting of elliptical Gaussians. Piner et al. (2010) find the eastern limb at a PA of $\sim 120^{\circ}$ and the western limb at a PA of $\sim 170^{\circ}$ during 2008 2009. They also perform the model fitting of circular Gaussians as we do, and identify components $\mathrm{C} 4$ and $\mathrm{C} 5$, which are located $0.58-0.72$ mas from the core at a PA of $137^{\circ}-154^{\circ}$ and $0.08-0.14$ mas at a PA of $-170^{\circ}--175^{\circ}$, respectively. The component $\mathrm{C} 2$ in our images could correspond to the component $\mathrm{C} 4$ in Piner et al. (2010), showing no detection of radial motion $(-0.01 \pm 0.01 c)$. The averaged PA of the components $\mathrm{C} 1, \mathrm{C} 2$, and $\mathrm{C} 3$ in our images is $\sim 156^{\circ}$, which is also comparable to the $\mathrm{PA}$ of the component $\mathrm{C} 4$. We find no emission at the location of the persistent western limb.

On the other hand, the characteristics of the NE component are different from those of the southeast jet components. It is 
Table 2. Gaussian models for the calibrated visibilities of VLBA $43 \mathrm{GHz}$ and GMVA $86 \mathrm{GHz}$ data.

\begin{tabular}{|c|c|c|c|c|c|}
\hline Epoch & Component & $\begin{array}{c}S \pm \sigma_{S}{ }^{a} \\
(\mathrm{mJy})\end{array}$ & $\begin{array}{r}r \pm \sigma_{r}^{b} \\
(\mathrm{mas})\end{array}$ & $\begin{array}{c}\mathrm{PA} \pm \sigma_{\mathrm{PA}}^{b} \\
(\mathrm{deg})\end{array}$ & $\begin{array}{r}a \pm \sigma_{a}^{c} \\
(\mathrm{mas})\end{array}$ \\
\hline \multirow[t]{5}{*}{2012 Feb. $12^{d}$} & Core & $188 \pm 19.4$ & $\ldots$ & $\ldots$ & $0.11 \pm 0.05$ \\
\hline & $\mathrm{NE}$ & $73 \pm 8.6$ & $0.14 \pm 0.01$ & $68.5 \pm 4.6$ & $0.18 \pm 0.02$ \\
\hline & $\mathrm{C} 3$ & $83 \pm 9.5$ & $0.15 \pm 0.03$ & $161.9 \pm 16.7$ & $0.04 \pm 0.09$ \\
\hline & $\mathrm{C} 2$ & $10 \pm 4.7$ & $0.54 \pm 0.05$ & $156.5 \pm 4.8$ & $0.21 \pm 0.05$ \\
\hline & $\mathrm{C} 1$ & $54 \pm 7.0$ & $1.13 \pm 0.11$ & $159.7 \pm 3.9$ & $0.73 \pm 0.22$ \\
\hline \multirow[t]{5}{*}{2012 Mar. $16^{d}$} & Core & $106 \pm 11.1$ & $\ldots$ & $\ldots$ & $0.09 \pm 0.06$ \\
\hline & $\mathrm{NE}$ & $98 \pm 11.1$ & $0.11 \pm 0.01$ & $51.8 \pm 6.4$ & $0.21 \pm 0.03$ \\
\hline & $\mathrm{C} 3$ & $77 \pm 8.4$ & $0.11 \pm 0.03$ & $156.4 \pm 15.8$ & $0.04 \pm 0.06$ \\
\hline & $\mathrm{C} 2$ & $24 \pm 4.1$ & $0.48 \pm 0.03$ & $155.8 \pm 3.6$ & $0.33 \pm 0.06$ \\
\hline & $\mathrm{C} 1$ & $43 \pm 5.4$ & $1.10 \pm 0.15$ & $156.0 \pm 7.4$ & $0.85 \pm 0.30$ \\
\hline \multirow[t]{5}{*}{2012 May $06^{d}$} & Core & $335 \pm 33.8$ & $\ldots$ & $\ldots$ & $0.17 \pm 0.06$ \\
\hline & $\mathrm{NE}$ & $15 \pm 5.1$ & $0.27 \pm 0.02$ & $20.1 \pm 3.9$ & $\ldots$ \\
\hline & $\mathrm{NE}^{\prime}$ & $25 \pm 5.4$ & $0.27 \pm 0.01$ & $109.1 \pm 3.1$ & $0.06 \pm 0.03$ \\
\hline & $\mathrm{C} 3$ & $50 \pm 6.9$ & $0.15 \pm 0.04$ & $167.5 \pm 14.5$ & $\ldots$ \\
\hline & $\mathrm{C} 1$ & $17 \pm 5.1$ & $1.11 \pm 0.04$ & $160.7 \pm 2.1$ & $0.14 \pm 0.08$ \\
\hline \multirow[t]{5}{*}{2012 Jun. $11^{d}$} & Core & $353 \pm 35.5$ & $\ldots$ & $\ldots$ & $0.14 \pm 0.07$ \\
\hline & $\mathrm{NE}$ & $15 \pm 3.9$ & $0.29 \pm 0.03$ & $45.6 \pm 5.0$ & $\ldots$ \\
\hline & $\mathrm{NE}^{\prime}$ & $11 \pm 3.8$ & $0.35 \pm 0.02$ & $105.9 \pm 2.8$ & $\ldots$ \\
\hline & $\mathrm{C} 2$ & $33 \pm 4.9$ & $0.45 \pm 0.03$ & $153.4 \pm 2.8$ & $0.34 \pm 0.05$ \\
\hline & $\mathrm{C} 1$ & $18 \pm 4.0$ & $1.17 \pm 0.05$ & $161.3 \pm 1.3$ & $0.26 \pm 0.14$ \\
\hline \multirow[t]{4}{*}{2013 Jan. $18^{d}$} & Core & $130 \pm 13.4$ & $\ldots$ & $\ldots$ & $0.06 \pm 0.05$ \\
\hline & $\mathrm{NE}$ & $88 \pm 9.4$ & $0.09 \pm 0.02$ & $19.6 \pm 11.6$ & $0.21 \pm 0.04$ \\
\hline & $\mathrm{C} 2$ & $17 \pm 3.7$ & $0.45 \pm 0.03$ & $150.6 \pm 4.2$ & $0.16 \pm 0.07$ \\
\hline & $\mathrm{C} 1$ & $21 \pm 3.9$ & $1.08 \pm 0.10$ & $154.5 \pm 0.10$ & $0.51 \pm 0.20$ \\
\hline \multirow[t]{4}{*}{2013 Feb. $15^{d}$} & Core & $148 \pm 15.2$ & $\ldots$ & $\ldots$ & $0.07 \pm 0.05$ \\
\hline & $\mathrm{NE}$ & $109 \pm 11.6$ & $0.12 \pm 0.02$ & $22.0 \pm 7.4$ & $0.26 \pm 0.03$ \\
\hline & $\mathrm{C} 2$ & $17 \pm 4.2$ & $0.42 \pm 0.03$ & $153.5 \pm 4.4$ & $0.16 \pm 0.06$ \\
\hline & $\mathrm{C} 1$ & $53 \pm 6.6$ & $1.05 \pm 0.25$ & $148.7 \pm 10.8$ & $0.97 \pm 0.04$ \\
\hline \multirow[t]{4}{*}{2012 May $19^{e}$} & Core & $235 \pm 71.4$ & $\ldots$ & $\ldots$ & $0.06 \pm 0.05$ \\
\hline & $\mathrm{NE}$ & $228 \pm 69.2$ & $0.11 \pm 0.02$ & $49.3 \pm 19.2$ & $0.67 \pm 0.04$ \\
\hline & SE & $23 \pm 12.8$ & $0.75 \pm 0.02$ & $155.8 \pm 3.3$ & $0.07 \pm 0.04$ \\
\hline & $\mathrm{SE}^{\prime}$ & $45 \pm 17.2$ & $1.28 \pm 0.02$ & $159.5 \pm 2.0$ & $0.17 \pm 0.05$ \\
\hline
\end{tabular}

Notes. ${ }^{(a)}$ Flux density and estimated errors. ${ }^{(b)} r$ and PAs are the polar coordinates of the component's center with respect to the core. The PAs are measured from north through east. $\sigma_{r}$ are estimated errors in the component position. $\sigma_{\mathrm{PA}}$ are estimated errors in the PA of the component. ${ }^{(c)}$ The FWHM of the radius of the circular Gaussian component and estimated errors in the component size. ${ }^{(d)}$ The VLBA $43 \mathrm{GHz}$ data. ${ }^{(e)}$ The GMVA $86 \mathrm{GHz}$ data.

difficult to perform model fitting using the relatively smooth emission distribution around the NE component. While the PA of the NE component changes by $\sim 50^{\circ}$ (from $\sim 70^{\circ}$ to $\sim 20^{\circ}$ ), the PAs of the southeast components are contained within a narrower range $\left(\sim 15^{\circ}\right)$. The separations between the NE component and the core for the third and fourth epochs are more than 0.1 mas farther than those for the other epochs, whose separations are slightly smaller than the minor axis of the original beam size. For the two epochs, there might be difficulties in separating the core and the NE component due to the apparent larger core sizes than the others. The absence of the long baselines (SC or MK) could be one of the reasons for the apparent larger core sizes, although the first epoch image can resolve the NE component from the core despite of the absence of SC baselines. Therefore, the apparent larger core sizes could be indeed intrinsic due to a certain change of the physical conditions in the jet, i.e., the Lorentz factor, the viewing angle, or the particle density in the jet. Piner et al. (2010) find the component C5 at a similar distance from the core ( $\sim 0.11$ mas $)$ in 2008 and 2009; however, its PA $\left(\sim-170^{\circ}\right)$ differs by more than $100^{\circ}$ from that of the NE component.

Figure 5 shows that the NE component does not follow any systematic motion. The scattering of the positions of the NE component is within $\sim 0.2$ mas. Except for the third and fourth epochs, it could show a position change of $\sim 0.1$ mas to the northwest (or slightly inward). Therefore, the characteristics of the NE component are different from those of regular PA swings of the innermost jets (e.g., in the quasar NRAO 150, Agudo et al. 2007; in the blazar OJ 287, Agudo et al. 2012).

We also note that Mrk 501 shows several PA changes out to the kiloparsec scale radio emission (Giroletti et al. 2008). The downstream jet PA changes are always in the clockwise direction $\left(\triangle \mathrm{PA}=\mathrm{PA}_{\text {out }}-\mathrm{PA}_{\text {in }}<0\right)$, while in this case, the rotation is in the opposite direction, i.e., $\Delta \mathrm{PA}=\mathrm{PA}_{\mathrm{C} 1}-\mathrm{PA}_{\mathrm{NE}}>0$. Thus, the PA of the NE component does not follow the "helical" trajectory of the outer jet, as opposed to the curved jet in 


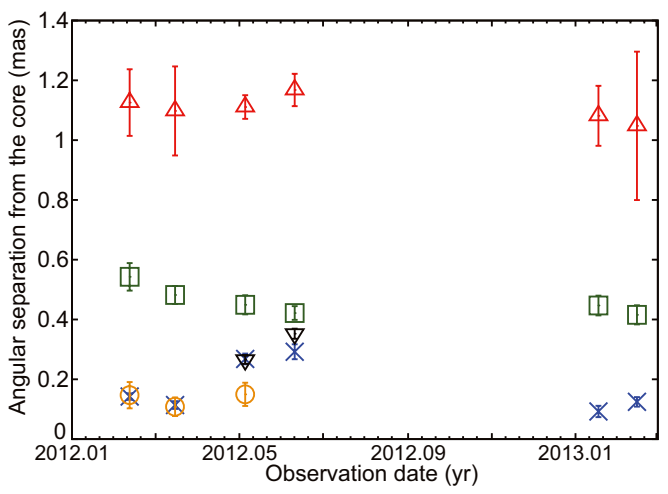

Fig. 3. Angular separation from the core to each component: C1 (blank red triangles), C2 (blank green squares), C3 (blank orange circles), NE (blue crosses), and $\mathrm{NE}^{\prime}$ (blank black inverted triangles).

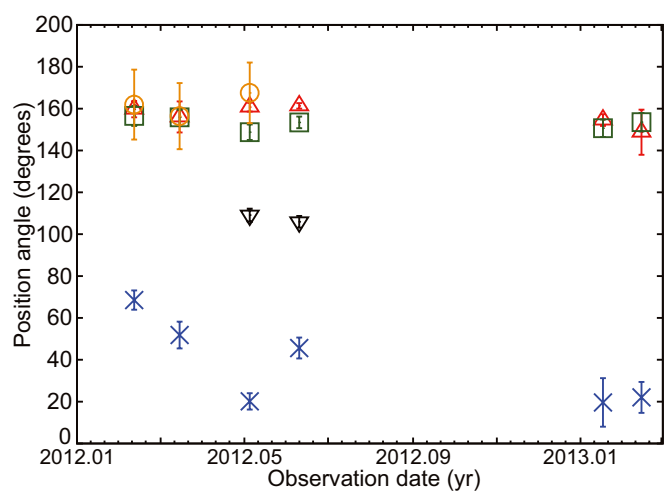

Fig. 4. Position angle of each component relative to the core. The same symbols as in Fig. 3 are used.

the blazar PKS 2136+141 (Savolainen et al. 2006). Similar jet PA mismatch between pc and kilo-pc scale is also observed in OJ 287 (Agudo et al. 2012). They suggest that different instability modes operate on the two spatial scales and at smaller scales at higher frequencies $(\geq 43 \mathrm{GHz})$ the instabilities grow rapidly in amplitude.

\subsubsection{Flux density variability}

From the results of our model-fitting, we study the evolution of the flux density for each component at $43 \mathrm{GHz}$. In Fig. 6, we plotted the flux of all the components. We perform a chi-square test of a flux variability for each component, and define a flare to be an increase in the VLBI flux that is $>3 \sigma$ of the averaged flux and greater than the noise level of the total flux density curve (e.g., Savolainen et al. 2002). As a result, there are no significant variations in the flux densities of the components $\mathrm{C} 2$ and $\mathrm{C} 3$. On the other hand, the flux densities of the component $\mathrm{C} 1$ are variable. For the 1st, 2nd, and 6th epochs, the flux densities of the component $\mathrm{C} 1$ are twice as high as those for the other epochs. These are more likely to be model-fit artifacts due to the larger Gaussian components on faint and extended emissions.

Chi-square tests using the model-fit results on the flux densities in the core and the NE component also suggest that they are variable. Although there are possible flux leakages between the closely spaced two components, when the core flux for the third and fourth epochs increased 150-250 mJy, the fluxes of the NE component decreased 60-90 mJy. Therefore, an increase in the core flux is still significant, such that there is a core flare

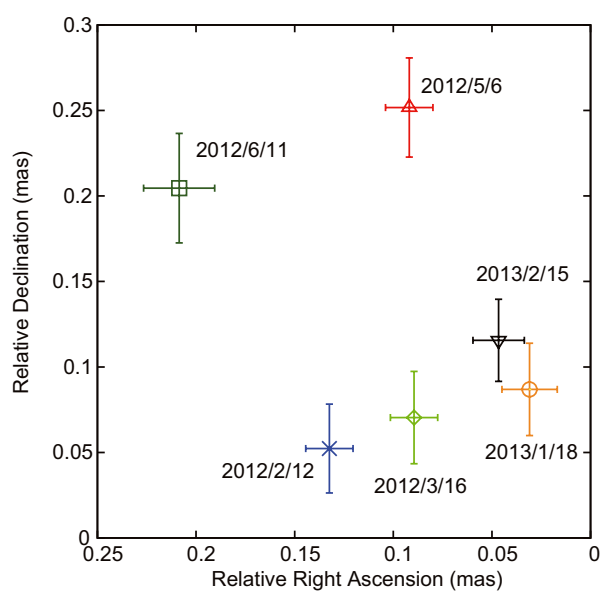

Fig. 5. Positions of the NE component relative to the core. The position of the core is set to $(0,0)$.

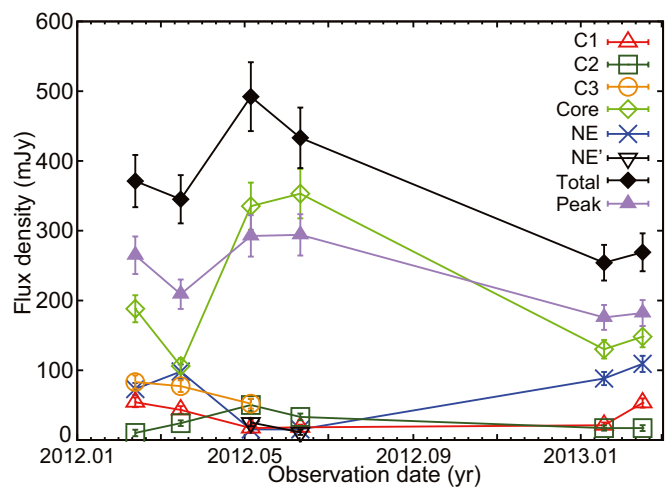

Fig. 6. Light curves of each component $\left(\mathrm{C} 1, \mathrm{C} 2, \mathrm{C} 3\right.$, Core, $\left.\mathrm{NE}, \mathrm{NE}^{\prime}\right)$, total CLEANed flux, and peak flux.

during these two epochs. This core flare could result from the larger core size, as is mentioned in Sect. 3.1.3. The total flux densities of two adjacent epochs $(1 \& 2,3 \& 4,5 \& 6)$ are consistent within the error bars. The total fluxes of the third and fourth epoch increased by $\geq 100 \mathrm{mJy}$, and the increase in the total fluxes is consistent with the increase in the core flux.

In order to confirm the core flare, we plotted the peak flux measured on images convolved with a circular beam of radius 0.3 mas, which is a conservative representation of the beam for the VLBA data at $43 \mathrm{GHz}$ (Blasi et al. 2013). For all epochs, the peak flux of the core with this circular beam contains $60-70 \%$ of the total flux density. For the third and fourth epochs, the peak flux is consistent with the core flux within the error bars. For the other epochs, most of the flux densities of the core and the $\mathrm{NE}$ component are collected within a radius of about 0.3 mas. Nevertheless, the peak fluxes are still variable, such that we confirm the core flare.

\subsubsection{Slice profiles}

To emphasize the existence of the NE component, we made a slice profile of the CLEAN image across the center of Gaussian models of the core and the NE component at each epoch using the $\mathcal{A I P S}$ task SLICE. In Fig. 7 we show all the slice profiles referenced to the Gaussian center of the core. Every slice profile is asymmetric and shows the presence of two humps corresponding to the core and the NE component. We fitted both single and double Gaussian component models to each slice profile using 


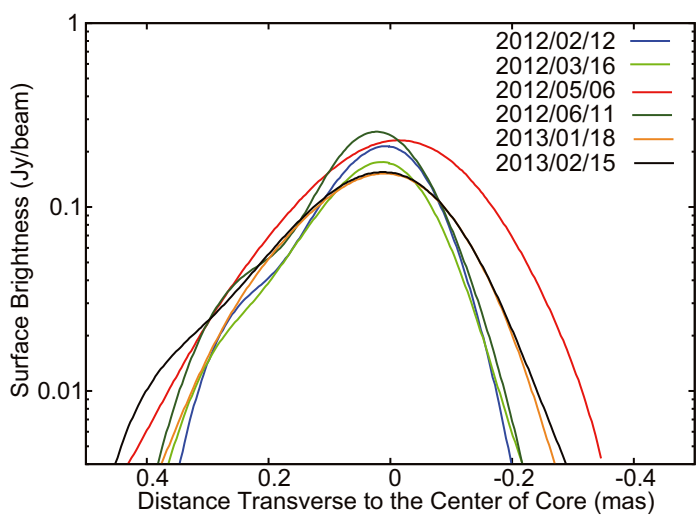

Fig. 7. Slice profiles of the brightness along the Gaussian center of the core and the NE component. Location of the Gaussian center is set to the reference position. All the profiles bulge eastward.

Table 3. Gaussian models for slice profiles of VLBA $43 \mathrm{GHz}$ images.

\begin{tabular}{ccccccc}
\hline \hline Epoch & Model $^{a}$ & ID & $\begin{array}{c}\text { Peak } \\
\left(\mathrm{mJy} \mathrm{beam}^{-1}\right)\end{array}$ & $\begin{array}{c}\text { Position } \\
(\mathrm{mas})\end{array}$ & $\begin{array}{c}F W H M \\
(\mathrm{mas})\end{array}$ & $\begin{array}{c}\mathrm{rms}^{b} \\
(\mathrm{mJy})\end{array}$ \\
\hline BK172A & 1 & Core & 208 & $\ldots$ & 0.20 & 158 \\
& 2 & Core & 207 & $\ldots$ & 0.17 & 25 \\
& 2 & NE & 39 & 0.16 & 0.21 & \\
BK172B & 1 & Core & 169 & $\ldots$ & 0.22 & 123 \\
& 2 & Core & 164 & $\ldots$ & 0.18 & 22 \\
& 2 & NE & 34 & 0.15 & 0.24 & \\
BK172C & 1 & Core & 227 & $\ldots$ & 0.31 & 93 \\
& 2 & Core & 215 & $\ldots$ & 0.27 & 29 \\
& 2 & NE & 36 & 0.18 & 0.30 & \\
BK172D & 1 & Core & 247 & $\ldots$ & 0.20 & 194 \\
& 2 & Core & 254 & $\ldots$ & 0.19 & 28 \\
& 2 & NE & 38 & 0.22 & 0.15 & \\
BK172E & 1 & Core & 150 & $\ldots$ & 0.28 & 70 \\
& 2 & Core & 137 & $\ldots$ & 0.23 & 16 \\
& 2 & NE & 40 & 0.16 & 0.24 & \\
BK172F & 1 & Core & 151 & $\ldots$ & 0.29 & 107 \\
& 2 & Core & 145 & $\ldots$ & 0.25 & 19 \\
& 2 & NE & 29 & 0.20 & 0.30 & \\
\hline
\end{tabular}

Notes. ${ }^{(a)}$ Fitting model type. Model 1 represents a single Gaussian model fitting. Model 2 represents a double Gaussian model fitting. ${ }^{(b)}$ Root sum squares of the residuals.

the $\mathcal{A I P S}$ task SLFIT. Table 3 lists the parameters of the fitted Gaussian models. By comparing the rms of the residuals for the single and double Gaussian fits, we determine that double Gaussian components provide a better fit. Although the parameters of the Gaussian models for the NE component are different from those in Table 2 owing to the difference of the dimensions, we confirm that there is clear emission at the northeast of the core for all epochs with a peak flux of $\sim 36 \mathrm{mJy} \mathrm{beam}^{-1}$ at the position of $\sim 0.18$ mas from the core and a FWHM of $\sim 0.24$ mas on average.

\subsection{The GMVA results}

In Fig. 8, we show the scaled GMVA image of Mrk 501 with Gaussian models. Visibility model-fitting in Difmap provides a reduced $\chi^{2}=0.86$ with this model. The result of model-fitting is summarized in Table 2. Owing to the lack of sensitivity on long baselines and sparse $(u, v)$ coverage, the visibility data are weighted using the natural weighting and tapered using a Gaussian taper with $\sigma_{\text {taper }}=1000 \mathrm{M} \lambda$. This yields a synthesized beam of $255 \mu$ as $\times 155 \mu$ as, which is about ten times larger than

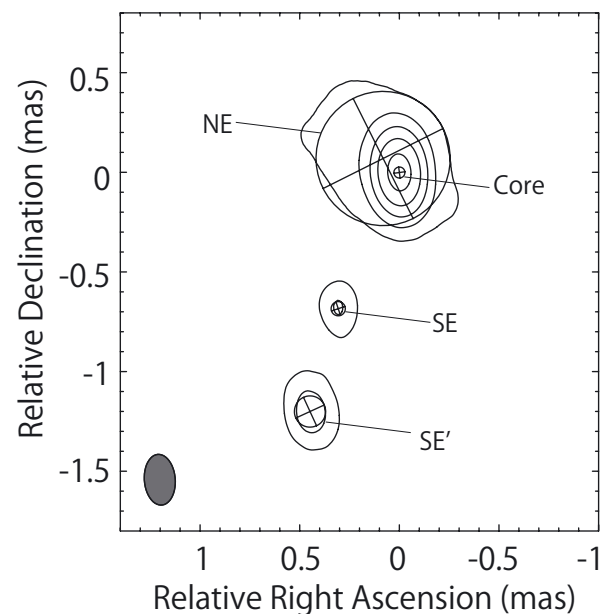

Fig. 8. GMVA image of Mrk 501 at $86 \mathrm{GHz}$ with four circular Gaussian model components with the restoring beam of $255 \mu$ as $\times 155 \mu$ as in PA $4.41^{\circ}$. The peak brightness is $235 \mathrm{mJy} \mathrm{beam}^{-1}$, and the contour levels are drawn at $(-1,1,2,4, \cdots) \times 10.7 \mathrm{mJy} \mathrm{beam}^{-1}$. The $1 \sigma$ noise level is $3.6 \mathrm{mJy}$ beam $^{-1}$.

the beam obtained by Giroletti et al. (2008), and comparable to the beam size in Fig. 2. We find an extended core emission modeled by a circular Gaussian, which is located 0.11 mas northeast of the core with $\sim 0.7$ mas in radius. Since the location of the Gaussian model is comparable to those of the NE component in $43 \mathrm{GHz}$ images, we label this component NE. We also find a jet knot (labeled SE) located at $(r, \theta)=\left(0.75\right.$ mas, $\left.156^{\circ}\right)$. This knot could correspond to the one detected by the GMVA at $86 \mathrm{GHz}$ in Giroletti et al. (2008), located at $(r, \theta)=\left(0.73\right.$ mas, $\left.172^{\circ}\right)$. The angular separation of the southeast knot from the core is comparable between these two epochs, which is consistent with the absence of superluminal jet motion. However, since there is a clear PA difference $\left(\sim 16^{\circ}\right)$, the SE component could be different from the knot in Giroletti et al. (2008). An extended jet knot $\left(\mathrm{SE}^{\prime}\right)$ located at $(r, \theta)=\left(1.28 \mathrm{mas}, 160^{\circ}\right)$ could correspond to component $\mathrm{C} 1$ in the $43 \mathrm{GHz}$ images.

Owing to low $\mathrm{S} / \mathrm{N}$ of the visibilities at transatlantic baselines, we set the upper limit of the unresolved core size as $55 \mu$ as $(0.037 \mathrm{pc})$ in radius, which is the deconvolved angular size of the core. Since we could not perform the amplitude self-calibration because of low $\mathrm{S} / \mathrm{N}$, we estimate the flux density uncertainties considering both a conservative flux calibration error of about $30 \%$ at $86 \mathrm{GHz}$ (Lee et al. 2008) and three times the map rms noise.

\subsection{VLBA $43 \mathrm{GHz}$ vs. GMVA $86 \mathrm{GHz}$}

\subsubsection{Brightness temperature}

We derive a lower limit of the brightness temperature in the core at $86 \mathrm{GHz}$ using an upper limit of the angular size and a lower limit of the flux. The brightness temperature for the core is estimated to be $T_{\mathrm{B}} \geq 4.5 \times 10^{9} \mathrm{~K}$. Although the flux of the core is more than three times as high as those of the previous $86 \mathrm{GHz}$ observations (Giroletti et al. 2008; Lee et al. 2008), the brightness temperature is comparable to their values owing to the larger deconvolved size. Therefore, it does not require a high Doppler factor at the base of the radio jet. For the $43 \mathrm{GHz}$ images, we derive the brightness temperature in the core using the deconvolved core size. The brightness temperatures in the core at $43 \mathrm{GHz}$ are estimated to be around a few $\times 10^{9} \mathrm{~K}$, which is 


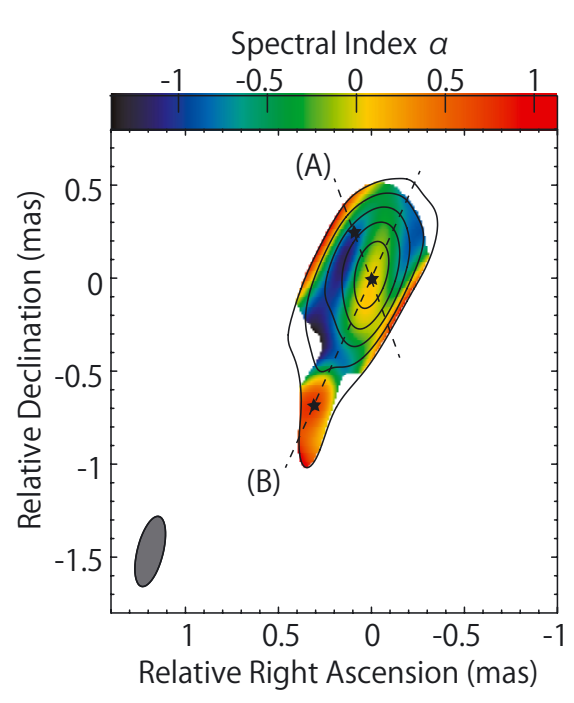

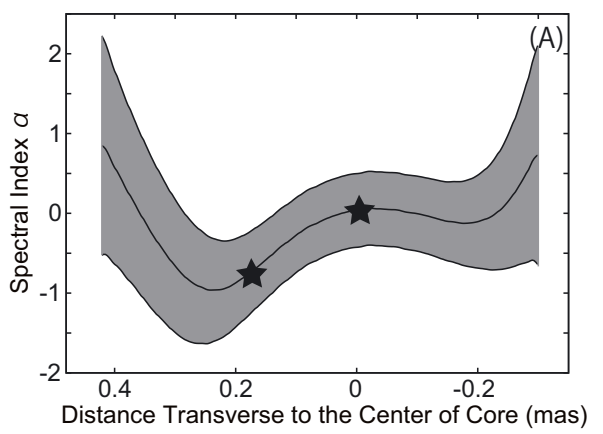

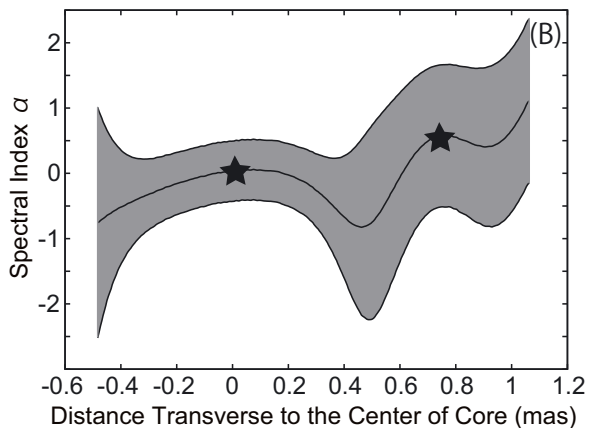

Fig. 9. Left panel: spectral index map between the third epoch VLBA $43 \mathrm{GHz}$ map and the GMVA $86 \mathrm{GHz}$ map. The contour map shows the VLBA $43 \mathrm{GHz}$ map, with the contour lev-

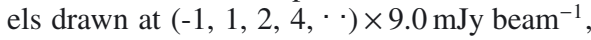
which is three times the rms noise of the offsource region of the $86 \mathrm{GHz}$ image. Black dotted lines show the locations of the slices and black stars indicate the centroid positions of the fitted Gaussian models. Right panel: A) Slice profile of spectral index map shown in Fig. 9 along Gaussian center of the core and the NE component at $\mathrm{PA}=20.1^{\circ}$ for the third epoch $43 \mathrm{GHz}$ observations. The gray area indicates $1 \sigma$ errors on the spectral index. The black stars show locations of the Gaussian models for the core and the NE component in the third epoch $43 \mathrm{GHz}$ map. B) Slice profile of spectral index map shown in Fig. 9 along Gaussian center of the core and the SE component at $\mathrm{PA}=155.8^{\circ}$ for the GMVA $86 \mathrm{GHz}$ observations. The black stars show locations of the Gaussian models for the core and the SE component in the $86 \mathrm{GHz}$ map. consistent with the values in Piner et al. (2010) at $43 \mathrm{GHz}$, and comparable to the $T_{\mathrm{B}}$ at $86 \mathrm{GHz}$.

The brightness temperatures of the NE component are $T_{\mathrm{B}} \sim$ $10^{8} \mathrm{~K}$ for VLBA $43 \mathrm{GHz}$ and $T_{\mathrm{B}} \geq 3 \times 10^{7} \mathrm{~K}$ for GMVA $86 \mathrm{GHz}$, which are much lower than that of the core and do not require a high Doppler factor.

\subsubsection{Spectral index}

In the left panel of Fig. 9, we show the spectral index distribution between the third epoch VLBA $43 \mathrm{GHz}$ image and the GMVA $86 \mathrm{GHz}$ image, which were obtained over a period of two weeks. We define the spectral index $\alpha$ as $S_{v} \propto v^{\alpha}$. The same $(u, v)$ range $(32-668 \mathrm{M} \lambda)$ and the same restoring beam size are used for the images of both frequencies. Then we produced the spectral index map by using the $\mathcal{A I P S}$ task COMB, clipping the pixels with three times the rms noise of the off-source region of the $86 \mathrm{GHz}$ image, referencing to the Gaussian center of the core for both images. Given the short time separation between these two images, we can obtain quasi-simultaneous spectral index maps. From this figure, we find the core region has a flat spectrum, the NE component has a flat-to-steep spectrum, and the southeast jet region has a flat-to-inverted spectrum. We made spectral index maps by taking into account the core shift effect ( $\sim 30 \mu$ as between $43 \mathrm{GHz}$ and $86 \mathrm{GHz}$, extrapolated from the power-law relation between the core position and frequency of Mrk 501 reported in Croke et al. 2010). However, the spectrum did not change significantly. Although we also made spectral index maps by using the $86 \mathrm{GHz}$ map without flux scaling, the spectral indices are consistent with the values with flux scaling within the error bars and the spectral tendency did not change.

Since the positions of Gaussian models between $43 \mathrm{GHz}$ and $86 \mathrm{GHz}$ do not coincide with each other, we estimate the value of the spectral indices using the slice profiles of the spectral index distribution (e.g., Hovatta et al. 2014). The uncertainties of the spectral index were calculated from the theory of the propagation of errors, including the frequency-dependent fluxdensity uncertainty and three times the map rms noise (e.g., Lico et al. 2012). The edges of the spectral index profiles have larger spectral uncertainties due to lower flux density than at the center of the profiles. The top right panel of Fig. 9 shows the spectral index profile along the Gaussian center of the core and the NE component position at the third epoch $43 \mathrm{GHz}$ observations $\left(\mathrm{PA}=20.1^{\circ}\right)$. The bottom right panel shows the spectral index profile along the Gaussian center of the core and the SE component position at the $86 \mathrm{GHz}$ observations $\left(\mathrm{PA}=155.8^{\circ}\right)$. From the spectral index profiles, we find that the spectral index of the core is $\alpha_{\mathrm{c}}=0.0 \pm 0.5$, that of the NE component is $\alpha_{\mathrm{NE}}=-0.8 \pm 0.5$, and that of the SE component is $\alpha_{\mathrm{SE}}=0.6 \pm 1.1$. The spectral index of the core is consistent with previous results $(\alpha \sim-0.5$ above the turnover, Giroletti et al. 2008; $\alpha \sim 0.2$ between $8 \mathrm{GHz}$ and $15 \mathrm{GHz}$, Hovatta et al. 2014). Compared with the core, the NE component has a steeper spectral index, and the SE component has a comparable or flatter spectral index. The flatter spectrum at the edges of the spectral profile in Fig. 9A is likely an artifact due to the large Gaussian size of the NE component the $86 \mathrm{GHz}$ image.

\section{Discussion}

\subsection{Possible origins of the NE component}

Since the NE component does not follow any systematic motion (Sect. 3.1.3), it can be regarded as a quasi-stationary component. Here we discuss two possible origins of the quasi-stationary component, which are internal shocks among discrete blobs and a part of dim continuous flow.

\subsubsection{Internal shocks}

One of the possible origins of the NE component is internal shocks. The internal shock model is one of the standard models for explaining blazar emission (e.g., Spada et al. 2001; Tanihata et al. 2003; Koyama et al. 2015). Based on the internal shock model, variation of Lorentz factors of the ballistic discrete ejecta makes the distance between the internal shocks and the central engine different. When we also take into account the effect of changing jet axes alignment as well, both the NE component and the radio core can be explained as the internal shock regions 


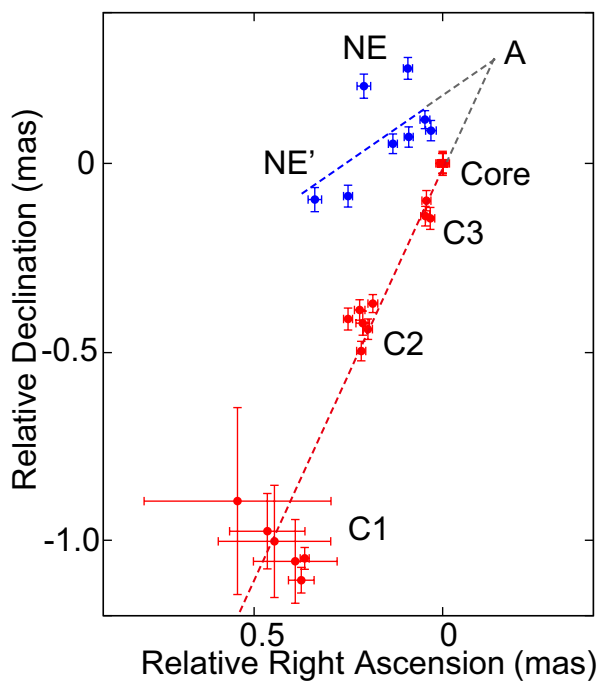

Fig. 10. Positions of the components $\mathrm{NE}$ and $\mathrm{NE}^{\prime}$ (Blue dots), and positions of the radio core, the components $\mathrm{C} 1, \mathrm{C} 2$, and $\mathrm{C} 3$ for all epochs (red dots). We define the dotted blue line (the least square line of the blue dots) as the eastern limb, and the dotted red line (that of the red dots) as the western limb. The intersection of the extrapolated lines (shown as the dotted grey lines) is labeled A. The position of the core is set to $(0,0)$.

along with different jet axes. Two jet axes are determined by least square fitting to the component positions for all the epochs in Fig. 10; one axis is fitted to the component NE and $\mathrm{NE}^{\prime}$ (eastern limb as the dotted blue line), and the other axis is fitted to the core, and the components $\mathrm{C} 1, \mathrm{C} 2$, and $\mathrm{C} 3$ (western limb as the dotted red line). In this case, the origin of the flow, which corresponds to the intersection of the two axes, is expected to be in the upstream of the core (Fig. 10, A). The angular separation between the intersection of the two axes and the NE component is 0.3 mas, or $2.8 \mathrm{pc}$ de-projected by assuming the jet viewing angle of $\sim 4^{\circ}$ (e.g., Giroletti et al. 2008). The distance between the central engine and the location of the internal shock region is approximately proportional to the square of the Lorentz factor of the ejecta (e.g., Spada et al. 2001). Hence, the quasi-stationary location of the internal shock regions can be explained by a small variation of Lorentz factors. In fact, the expected distance from the central engine to the NE component is comparable to the distance to the core in Koyama et al. (2015) where we explained that a small variation of Lorentz factors in its quiescent state makes the spatial distribution of the location of the internal shocks within 0.2 mas. Therefore, internal shocks can be one of the possible origins of the quasi-stationary NE component.

It is also worth discussing what will happen in its active state. When the variation of the Lorentz factor is significant in its active state, the location of the internal shock region would change considerably. In the case of another nearby TeV blazar Mrk 421, we detected significant core wandering up to 0.5 mas soon after its big X-ray flare (Niinuma et al. 2015). If the radio core wanders $\sim 0.5$ mas when Mrk 501 is in active state, the location of the NE component would spread widely, or it might show systematic motion. We need further constraints on the core position by using astrometric observations to study the exact location of the NE component.

\subsubsection{A part of dim flow}

Another possible origin of the NE component is a part of dim continuous flow, which is usually below the detection threshold of VLBA at $43 \mathrm{GHz}$. Under certain conditions, such as the injection of radio-emitting plasma at the base of the jet related to a high-energy flare, or a slight increase in Doppler factors, the observed flux can be enhanced ${ }^{4}$. Then the enhanced emission can be above the detection threshold. The possible flux variability of the NE component (Sect. 3.1.4) may support this scenario. Although there are possible flux leakages between the core and the NE component, the moderate variability (from $15 \mathrm{mJy}$ to $109 \mathrm{mJy}$ over $\sim 8$ months with the size of 0.26 mas) suggests a low value of "variability Doppler factor" 5 as $\delta \sim 3$. This value is consistent with the results obtained by low brightness temperatures obtained in Sect. 3.3.1. The location of the NE component is quasi-stationary within $\sim 0.2$ mas over one year, which puts the upper limit of its apparent velocity as $\beta_{\text {app }} \lesssim 0.4$

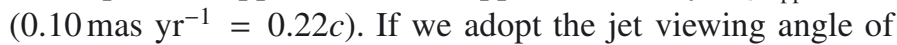
$\theta \sim 4^{\circ}$ (e.g., Giroletti et al. 2004), the corresponding Lorentz factor of the emission region is estimated to be $\Gamma \lesssim 2$ (a Doppler factor of $\delta \lesssim 4$ ). Since the obtained upper limit of the Lorentz factor is lower than the typical values of one-zone SED modeling (e.g., $\delta \sim \Gamma \sim 10$ : Bartoli et al. 2012), the NE component is not completely identical to the high-energy emission region. Such a low value of the Lorentz factor may support the idea that the NE component corresponds to the slower outer layer.

Finally, we discuss the origin of the dim flow. The origin of the dim flow can be the core or the intersection of the two axes (position A shown in Fig. 10). If the origin of the jet is located at the core, the jet should be initially ejected toward the northeast direction from the core rather than the persistent southeast jet, then it apparently bends $\sim 90^{\circ}$ toward the southeast. New component emergence at an unusual PA similar to Mrk 501 has been reported in the quasar 3C 279; however, it does not show quasistationary behavior like the NE component in Mrk 501 but shows an outward motion (Lu et al. 2013). Such an unusual jet PA could be similar to the erratic PA changes of innermost jets, which is possibly caused by hydrodynamic instabilities due to asymmetric jet injection (e.g., McKinney \& Blandford 2009; Aloy et al. 2003), as observed in a few blazars (e.g., OJ 287: Agudo et al. 2012, BL Lac: Cohen et al. 2014). However, it is difficult to bend the jet apparently $\sim 90^{\circ}$, that is, intrinsically $\sim 40^{\circ}$ (for $\theta \sim 4^{\circ}$ ) (e.g., via oblique shocks: Tingay 1997). Therefore, the upstream of the core (position A in Fig. 10) is more likely to be the jet origin, and a slight change in the jet direction toward observers at the NE component can enhance the Doppler factor and the flux of the dim flow (e.g., Gomez et al. 1993).

\subsection{Spectral index of southeast jet feature}

Our observation could not provide strong constraints on the spectral index of the jet feature because of the limited capability of the array. To obtain more precise GMVA images, we need better $(u, v)$-coverage to be provided by the addition of new stations to the array. Recently, the Korean VLBI Network (Lee et al. 2014) joined GMVA observations (Hodgson et al. 2014), which will increase the number of east-west baselines when there is no common sky between Europe and the USA. The east-west baselines of $\sim 9000 \mathrm{~km}$ is crucial for resolving the core and the NE component with typically $50 \mu$ as resolution. Filling short baseline spacings will also enable us to perform

4 If the enhanced flux of the NE component is related to a high-energy flare, it could be a $\gamma$-ray flare in 2011 October (Bartoli et al. 2012) (Sect. 3.1.1).

5 Defined by Jorstad et al. (2005) by assuming that the variability timescale corresponds to the light-travel time across the knot. 
more accurate calibration. Thus, the increased number of the baselines is important for obtaining images and spectral index maps at higher fidelity.

Between 8 and $15 \mathrm{GHz}$, the spectral index at the component 0.6 mas southeast of the core of Mrk 501 is $\alpha=-0.5 \pm 0.1$, and the mean spectral index of the overall jets of BL Lac objects is $\alpha \sim-0.8$ (Hovatta et al. 2014). They also point out that the jet spectrum flattens on average by $\sim 0.2$ at the location of the jet components, indicating the enhancement of particle density due to shocks in the jets (Mimica et al. 2009), or superposition of multiple components along the line of sight. A flatto-inverted spectral distribution is also seen at the component $\mathrm{C} 4$, located 0.6 mas southeast of the core, having $\alpha \sim 0.2$ between $1.6 \mathrm{GHz}$ and $4.8 \mathrm{GHz}$ space VLBI observations (Giroletti et al. 2004), while the other components have a steep spectrum. Edwards et al. (2000) also suggest that it could be connected to the inner spine flat-spectrum region. If the SE component is a part of the inner spine, the location of the component could change $\sim 0.3$ mas within the separation period between $43 \mathrm{GHz}$ and $86 \mathrm{GHz}$ observations (13 days), by applying $\theta_{\mathrm{c}}=4^{\circ}$ and $\Gamma_{\mathrm{c}}=17$, so that it leads to large image misalignment. Among other sources, a flat-to-inverted spectral distribution is seen in one of the index maps of the jet in the quasar NRAO 150 between $43 \mathrm{GHz}$ and $86 \mathrm{GHz}$ (Molina et al. 2014), but the separation of four months between the two images may cause large image misalignment due to structure change. Since such a flat-to-inverted jet spectrum is quite unusual and has not yet been examined precisely, it is important to confirm whether it is real by performing simultaneous observations between 43 and $86 \mathrm{GHz}$.

\section{Conclusions}

We have explored the sub-pc-to-pc scale region in Mrk 501 using the VLBA at $43 \mathrm{GHz}$ and the GMVA at $86 \mathrm{GHz}$ between 2012-2013.

1. We find a new off-axis jet structure, the NE component, located northeast of the core. The position angle of the NE component changes from $70^{\circ}$ to $20^{\circ}$, while those for the southeast jet components reside within $\sim 15^{\circ}$. The scattering of the positions of the NE component relative to the core is within $\sim 0.2$ mas for all of our VLBA $43 \mathrm{GHz}$ observations. There is a small core flare during the third and fourth epochs, and it could be due to the large core size. From the slice profiles, the component NE is located at 0.18 mas with the peak flux of $36 \mathrm{mJy}^{-1}$ beam $^{-1}$ and FWHM of 0.24 mas on average.

2. We obtain the quasi-simultaneous GMVA $86 \mathrm{GHz}$ image within two weeks of the third epoch of the VLBA $43 \mathrm{GHz}$ observations. Although the visibilities on transatlantic baselines show weak coherence and low signal-to-noise, we detect the northeast feature located at 0.11 mas from the core, and the southeast jet feature located at 0.75 mas and 1.28 mas from the core.

3 . From the spectral index profile analysis between the quasisimultaneous 43 and $86 \mathrm{GHz}$ images, the spectral index of the core is flat and consistent with previous results, while that of the NE component is flat-to-steep, and that of the SE component is flat-to-inverted. To confirm these values, simultaneous high-frequency observations with more eastwest baselines are necessary.

4. Since the NE component can be regarded a quasi-stationary component, internal shocks or a part of dim flow could be possible origins of the component. Determination of the more precise location of the NE component using radio core astrometry, and higher sensitivity observations toward the dim flow are needed.

Acknowledgements. We thank the anonymous referee for useful comments and suggestions. The National Radio Astronomy Observatory is a facility of the National Science Foundation operated under cooperative agreement by Associated Universities, Inc. This work made use of the Swinburne University of Technology software correlator (Deller et al. 2011), developed as part of the Australian Major National Research Facilities Programme and operated under license. This paper is partially based on observations carried out with the VLBA, the MPIfR $100 \mathrm{~m}$ Effelsberg Radio Telescope, the IRAM Plateau de Bure Millimetre Interferometer, the IRAM $30 \mathrm{~m}$ Millimeter Telescope, the Onsala $20 \mathrm{~m}$ Radio Telescope, and the Metsähovi $14 \mathrm{~m}$ Radio Telescope. IRAM is supported by MPG (Germany), INSU/CNRS (France), and IGN (Spain). The GMVA is operated by the MPIfR, IRAM, NRAO, OSO, and MRO. We thank the staff of the participating observatories for their efficient and continuous support. S.K. acknowledges this research grant provided by the Global COE program of University of Tokyo. Part of this work was done with the contribution of the Italian Ministry of Foreign Affairs and University and Research for the collaboration project between Italy and Japan. This work was partially supported by Grant-in-Aid for Scientific Research, KAKENHI 24340042 (A.D.) and 2450240 (M.K.) from the Japan Society for the Promotion of Science (JSPS). E.R. and M.A.P.T. acknowledge partial support from the Spanish MINECO through projects AYA-2012-38491-C02-01 and AYA-2012-38491-C02-02. E.R. also acknowledges partial support from the Generalitat Valenciana through project PROMETEOII/2014/057. S.K. is thankful to Jeffrey A. Hodgson and Bindu Rani for useful discussion and comments on the paper.

\section{References}

Agudo, I., Bach, U., Krichbaum, T. P., et al. 2007, A\&A, 476, L17 Agudo, I., Marscher, A. P., Jorstad, S. G., et al. 2012, ApJ, 747, 63 Aloy, M.-Á., Martí, J.-M., Gómez, J.-L., et al. 2003, ApJ, 585, L109 Bartoli, B., Bernardini, P., Bi, X. J., et al. 2012, ApJ, 758, 2

Begelman, M. C., Blandford, R. D., \& Rees, M. J. 1984, Rev. Mod. Phys., 56, 255

Blasi, M. G., Lico, R., Giroletti, M., et al. 2013, A\&A, 559, A75

Cohen, M. H., Meier, D. L., Arshakian, T. G., et al. 2014, ApJ, 787, 151

Croke, S. M., O’Sullivan, S. P., \& Gabuzda, D. C. 2010, MNRAS, 402, 259

Deller, A. T., Brisken, W. F., Phillips, C. J., et al. 2011, PASP, 123, 275

Edwards, P. G., Giovannini, G., Cotton, W. D., et al. 2000, PASJ, 52, 1015

Giroletti, M., Giovannini, G., Feretti, L., et al. 2004, ApJ, 600, 127

Giroletti, M., Giovannini, G., Cotton, W. D., et al. 2008, A\&A, 488, 905

Gomez, J. L., Alberdi, A., \& Marcaide, J. M. 1993, A\&A, 274, 55

Hodgson, J. A., Krichbaum, T. P., Marscher, A. P., et al. 2014, ArXiv e-prints [arXiv: 1407.8112]

Homan, D. C., Kadler, M., Kellermann, K. I., et al. 2009, ApJ, 706, 1253

Homan, D. C., Lister, M. L., Kovalev, Y. Y., et al. 2015, ApJ, 798, 134

Hovatta, T., Aller, M. F., Aller, H. D., et al. 2014, AJ, 147, 143

Jorstad, S. G., Marscher, A. P., Lister, M. L., et al. 2005, AJ, 130, 1418

Kellermann, K. I., Vermeulen, R. C., Zensus, J. A., \& Cohen, M. H. 1998, AJ 115,1295

Komatsu, E., Dunkley, J., Nolta, M. R., et al. 2009, ApJS, 180, 330

Koyama, S., Kino, M., Doi, A., et al. 2015, PASJ, 67, 67

Lee, S.-S., Lobanov, A. P., Krichbaum, T. P., et al. 2008, AJ, 136, 159

Lee, S.-S., Petrov, L., Byun, D.-Y., et al. 2014, AJ, 147, 77

Lico, R., Giroletti, M., Orienti, M., et al. 2012, A\&A, 545, A117

Lister, M. L., Cohen, M. H., Homan, D. C., et al. 2009, AJ, 138, 1874

Lister, M. L., Aller, M. F., Aller, H. D., et al. 2013, AJ, 146, 120

Lu, R.-S., Fish, V. L., Akiyama, K., et al. 2013, ApJ, 772, 13

McKinney, J. C., \& Blandford, R. D. 2009, MNRAS, 394, L126

Mimica, P., Aloy, M.-A., Agudo, I., et al. 2009, ApJ, 696, 1142

Molina, S. N., Agudo, I., Gómez, J. L., et al. 2014, A\&A, 566, A26

Niinuma, K., Kino, M., Doi, A., et al. 2015, ApJ, 807, L14

Orienti, M., Venturi, T., Dallacasa, D., et al. 2011, MNRAS, 417, 359

Piner, B. G., Pant, N., Edwards, P. G., \& Wiik, K. 2009, ApJ, 690, L31

Piner, B. G., Pant, N., \& Edwards, P. G. 2010, ApJ, 723, 1150

Rani, B., Krichbaum, T. P., Marscher, A. P., et al. 2015, A\&A, 578, A123

Savolainen, T., Wiik, K., Valtaoja, E., Jorstad, S. G., \& Marscher, A. P. 2002, A\&A, 394, 851

Savolainen, T., Wiik, K., Valtaoja, E., et al. 2006, ApJ, 647, 172

Shepherd, M. C. 1997, in Astronomical Data Analysis Software and Systems VI, eds. G. Hunt, \& H. Payne, ASP Conf. Ser., 125, 77

Spada, M., Ghisellini, G., Lazzati, D., \& Celotti, A. 2001, MNRAS, 325, 1559 Tanihata, C., Takahashi, T., Kataoka, J., \& Madejski, G. M. 2003, ApJ, 584, 153 Tingay, S. J. 1997, A\&A, 327, 550 